\title{
Existence of Solutions for Sturm-Liouville Boundary Value Problem of Impulsive Differential Equations
}

\author{
Hong-Rui Sun, ${ }^{1}$ Ya-Ning Li, ${ }^{1}$ Juan J. Nieto, ${ }^{2,3}$ and Qing Tang ${ }^{2}$ \\ ${ }^{1}$ School of Mathematics and Statistics, Lanzhou University, Gansu, Lanzhou 730000, China \\ ${ }^{2}$ Departamento de Análisis Matemático, Universidad de Santiago de Compostela, \\ Santiago de Compostela 15782, Spain \\ ${ }^{3}$ Department of Mathematics, Faculty of Science, King Abdulaziz University, P.O. Box 80203, \\ Jeddah 21589, Saudi Arabia \\ Correspondence should be addressed to Hong-Rui Sun, hrsun@lzu.edu.cn
}

Received 20 January 2012; Revised 13 March 2012; Accepted 3 April 2012

Academic Editor: D. O’Regan

Copyright (C 2012 Hong-Rui Sun et al. This is an open access article distributed under the Creative Commons Attribution License, which permits unrestricted use, distribution, and reproduction in any medium, provided the original work is properly cited.

This paper is concerned with the existence of solutions for Sturm-Liouville boundary value problem of a class of second-order impulsive differential equations, under different assumptions on the nonlinearity and impulsive functions, existence criteria of single and multiple solutions are established. The main tools are variational method and critical point theorems. Some examples are also given to illustrate the main results.

\section{Introduction}

Impulsive differential equation is one of the main tools to study the dynamics of processes in which sudden changes occur. The theory of impulsive differential equation has recently received considerable attention, see [1-9]. Some classical tools such as fixed-point theorems in cones and the method of upper and lower solutions with monotone iterative technique have been widely used to study impulsive differential equations. In the last few years, variational method has been used to determine the existence of solutions for impulsive differential equation possessing a variational structure under certain boundary condition, one can refer to [10-26] and the references therein for detailed discussions.

Especially, in [12], Nieto and O'Regan studied the nonlinear Dirichlet impulsive problem:

$$
-u^{\prime \prime}(t)+u(t)=f(t, u(t)), \quad t \in[0, T] \backslash\left\{t_{1}, t_{2}, \ldots, t_{p}\right\},
$$




$$
\begin{gathered}
\Delta u^{\prime}\left(t_{j}\right)=I_{j}\left(u\left(t_{j}^{-}\right)\right), \quad j=1,2, \ldots, p, \\
u(0)=u(T)=0,
\end{gathered}
$$

where $f \in C([0, T] \times \mathbb{R}, \mathbb{R}), I_{j} \in C(\mathbb{R}, \mathbb{R})$, and $j=1,2, \ldots, p$. By the least action principle, the existence of a solution was obtained by assuming sublinear growth on the nonlinearity and impulses.

In [26], Zhou and Li discussed the problem:

$$
-u^{\prime \prime}(t)+g(t) u(t)=f(t, u(t)), \quad t \in[0, T] \backslash\left\{t_{1}, t_{2}, \ldots, t_{p}\right\},
$$

with impulse conditions (1.2), and Dirichlet boundary condition (1.3), where $f \in C([0, T] \times$ $\mathbb{R}, \mathbb{R})$ and $g \in L^{\infty}([0, T], \mathbb{R})$. By using a symmetric Mountain Pass theorem, the existence result of an infinite number of solutions was obtained.

Tian and Ge in [20] studied the existence of multiple solutions for the following equation with impulsive effect:

$$
\begin{gathered}
-\left(p(t) u^{\prime}\right)^{\prime}+q(t) u=f(t, u), \quad t \in[0,1] \backslash\left\{t_{1}, t_{2}, \ldots, t_{p}\right\}, \\
-\Delta\left(p\left(t_{j}\right) u\left(t_{j}\right)\right)=I_{j}\left(u\left(t_{j}\right)\right), \quad j=1,2, \ldots, p, \\
\alpha u(0)-\beta u^{\prime}(0)=0, \quad \gamma u(1)+\sigma u^{\prime}(1)=0 .
\end{gathered}
$$

By applying variational methods and upper and lower solutions methods, they obtained the existence of at least four solutions and gave some accurate characteristics of the solutions. In the special case of $p(t) \equiv 1, q(t) \equiv \lambda$ and $I_{j}\left(u\left(t_{j}\right)\right)=d_{j}$, in [19] they studied the corresponding linear and nonlinear problem and established some existence results of positive solutions via critical point theory and variational methods. In [13], Sun and Chen considered the equation:

$$
-\left(p(t) u^{\prime}\right)^{\prime}+r(t) u^{\prime}+q(t) u=f(t, u), \quad t \in[0,1] \backslash\left\{t_{1}, t_{2}, \ldots, t_{p}\right\},
$$

with impulsive condition (1.6) and Neumann boundary condition $u^{\prime}\left(0^{+}\right)=u^{\prime}\left(1^{-}\right)=0$, they got existence criteria of at least one solution, two solutions and infinitely many solutions under some different conditions.

In the above-cited articles, analogous results are also given when the impulses are absent, so they cannot reflect the impact of the impulses on the existence of the solutions. In [24], Zhang and Li studied the periodic solutions generated by impulses for a second-order impulsive differential equation. As defined in [24], a solution is called a solution generated by impulses if this solution is nontrivial only if impulsive terms are not zero.

Impulsive effects exist widely in many evolution processes in which their states are changed abruptly at certain moments of time. Many systems involve the impulsive condition which depends not only on the derivative of a function but also the function itself. A few papers discussed the solutions of impulsive differential equations involving impulses both on the function and on its derivative by critical point theory; see for example [21, 22]. 
Inspired by the above results, in this paper we consider the existence and multiplicity of solution to the following Sturm-Liouville boundary value problem of a class of secondorder impulsive differential equations:

$$
\begin{gathered}
-u^{\prime \prime}(t)+u(t)=f(t, u(t)), \quad t \in[0, T] \backslash\left\{t_{1}, t_{2}, \ldots, t_{p}\right\}, \\
-\Delta u\left(t_{j}\right)=A_{j} u\left(t_{j}^{-}\right), \quad j=1,2, \ldots, p, \\
-\Delta u^{\prime}\left(t_{j}\right)=I_{j}\left(u\left(t_{j}^{-}\right)\right)-A_{j} u^{\prime}\left(t_{j}^{+}\right), \quad j=1,2, \ldots, p, \\
\alpha u(0)-\beta u^{\prime}(0)=0, \quad \gamma u(T)+\sigma u^{\prime}(T)=0,
\end{gathered}
$$

where $f \in C([0, T] \times \mathbb{R}, \mathbb{R}), \alpha, \beta, \gamma, \sigma \geq 0, \alpha+\beta>0, \gamma+\sigma>0 . p \in \mathbb{N}, 0=t_{0}<t_{1}<t_{2}<\cdots<t_{p}<$ $t_{p+1}=T, \Delta u\left(t_{j}\right)=u\left(t_{j}^{+}\right)-u\left(t_{j}^{-}\right), u\left(\mathrm{t}_{j}^{+}\right)$(or $\left.u\left(t_{j}^{-}\right)\right)$denotes the right limit (or left limit) of $u(t)$ at $t=t_{j}, \Delta u^{\prime}\left(t_{j}\right)=u^{\prime}\left(t_{j}^{+}\right)-u^{\prime}\left(t_{j}^{-}\right), u^{\prime}\left(t_{j}^{+}\right)$(or $\left.u^{\prime}\left(t_{j}^{-}\right)\right)$denotes the right limit (or left limit) of $u^{\prime}(t)$ at $t=t_{j}, A_{j} \in \mathbb{R}, I_{j} \in C(\mathbb{R}, \mathbb{R}), j=1,2, \ldots, p$. For convenience, we denote (1.9)-(1.12) as problem $(P)$.

We begin by establishing the corresponding variational framework of problem $(P)$ in an appropriate space of functions. Then under the assumption that the nonlinearity and the impulsive functions are superlinear, we get the existence of at least one nontrivial solution. Furthermore, when $f$ is odd about the second variable and $I_{j}$ are odd, the existence of an infinite number of solutions is obtained. Moreover, we study the existence of solutions generated by impulses. Under suitable hypothesis, existence criteria of at least one solution and infinitely many solutions generated by impulses of problem $(P)$ are established. The main tools are the Mountain Pass theorem, symmetric Mountain Pass theorem, and the least action principle.

Note that when $A_{j}=0$, the impulsive conditions (1.10) and (1.11) of problem $(P)$ degenerate to the impulsive condition (1.2), and when $\beta=\sigma=0$, (1.12) is Dirichlet boundary condition; however, our assumptions on $f$ are different from the assumptions on $f$ in [12]. The construction of a suitable space of functions and corresponding energy functional become more complicated with the impulse effects of (1.10), (1.11), and Sturm-Liouville boundary conditions taken into consideration.

The rest of this paper is organized as follows. In Section 2, we list some preliminary results and several lemmas, which are important in proving the existence of solutions. In Section 3, we state and prove the main results and give some examples to illustrate them.

\section{Preliminary}

We define the space:

$$
\begin{aligned}
X=\left\{u \in L^{2}(0, T) \mid u_{\mid\left(t_{j}, t_{j+1}\right)} \in H^{1}\left(t_{j}, t_{j+1}\right), j=0,1, \ldots, p,\right. & \\
& \left.-\Delta u\left(t_{j}\right)=A_{j} u\left(t_{j}^{-}\right), j=1, \ldots, p, u(0)=0 \text { if } \beta=0, u(T)=0 \text { if } \sigma=0\right\} .
\end{aligned}
$$


Its norm is induced by the inner product:

$$
(u, v)=\int_{0}^{T}\left[u^{\prime}(t) v^{\prime}(t)+u(t) v(t)\right] d t, \quad u, v \in X
$$

that is,

$$
\|u\|=\left(\int_{0}^{T}\left[\left(u^{\prime}(t)\right)^{2}+u^{2}(t)\right] d t\right)^{1 / 2}, \quad u \in X .
$$

If $u \in X$, then $u_{\mid\left(t_{j}, t_{j+1}\right)} \in H^{1}\left(t_{j}, t_{j+1}\right)$, and thus the limits $u\left(t_{j}^{+}\right)$and $u\left(t_{j+1}^{-}\right)$exist, we define

$$
u^{j}(t)= \begin{cases}u(t), & t \in\left(t_{j}, t_{j+1}\right), \\ u\left(t_{j}^{+}\right), & t=t_{j}, \\ u\left(t_{j+1}^{-}\right), & t=t_{j+1},\end{cases}
$$

then $u^{j} \in C\left[t_{j}, t_{j+1}\right]$.

Now we state some lemmas, which are needed in the proof of the main results.

Lemma 2.1. $X$ is a Hilbert space.

Proof. Clearly, $X$ is a linear space equipped with the inner product (2.2), so $X$ is an inner space. Next, we will show that $X$ is complete.

Suppose $\left\{u_{n}\right\} \subset X$ is an arbitrary Cauchy sequence, from the definition of $X$, for $j=0,1, \ldots, p,\left\{u_{n}^{j}\right\}$ is also a Cauchy sequence in $H^{1}\left(t_{j}, t_{j+1}\right)$. Then, there exists $u^{j} \in H^{1}\left(t_{j}, t_{j+1}\right)$ with $\lim _{n \rightarrow \infty} u_{n}^{j}=u^{j}$ in $H^{1}\left(t_{j}, t_{j+1}\right)$; therefore, $\left\{u_{n}^{j}\right\}$ converges to $u^{j}$ in $C\left[t_{j}, t_{j+1}\right]$, that is, $\left\|u_{n}^{j}-u^{j}\right\|_{C\left[t_{j}, t_{j+1}\right]} \rightarrow 0$ as $n \rightarrow \infty$. So we have

$$
\begin{aligned}
u^{j}\left(t_{j}\right) & =\lim _{n \rightarrow \infty} u_{n}^{j}\left(t_{j}\right)=\lim _{n \rightarrow \infty} u_{n}\left(t_{j}^{+}\right)=\lim _{n \rightarrow \infty}\left(1-A_{j}\right) u_{n}\left(t_{j}^{-}\right) \\
& =\lim _{n \rightarrow \infty}\left(1-A_{j}\right) u_{n}^{j-1}\left(t_{j}\right)=\left(1-A_{j}\right) u^{j-1}\left(t_{j}\right),
\end{aligned}
$$

that is,

$$
\left(1-A_{j}\right) u^{j-1}\left(t_{j}\right)=u^{j}\left(t_{j}\right)
$$


Set

$$
u(t)= \begin{cases}u^{j}(t), & t \in\left(t_{j}, t_{j+1}\right), j=0, \ldots, p, \\ u^{0}\left(0^{+}\right), & t=0, \\ u^{p}\left(T^{-}\right), & t=T .\end{cases}
$$

Then we have

$$
\left(1-A_{j}\right) u\left(t_{j}^{-}\right)=\left(1-A_{j}\right) u^{j-1}\left(t_{j}\right)=u^{j}\left(t_{j}\right)=u\left(t_{j}^{+}\right)
$$

So $-\Delta u\left(t_{j}\right)=A_{j} u\left(t_{j}^{-}\right)$and $u \in X$.

Observe that

$$
\begin{aligned}
\left\|u_{n}-u\right\|^{2} & =\sum_{j=0}^{p} \int_{t_{j}}^{t_{j+1}}\left|\left(u_{n}^{j}\right)^{\prime}(t)-\left(u^{j}\right)^{\prime}(t)\right|^{2}+\left|u_{n}^{j}(t)-u^{j}(t)\right|^{2} d t \\
& =\sum_{j=0}^{p}\left\|u_{n}^{j}-u^{j}\right\|_{H^{1}\left(t_{j}, t_{j+1}\right)^{\prime}}^{2}
\end{aligned}
$$

since $\left\{u_{n}^{j}\right\}$ converge to $u^{j}$ in $H^{1}\left(t_{j}, t_{j+1}\right)$, then the Cauchy sequence $\left\{u_{n}\right\}$ converge to $u$ in $X$, that is, $X$ is complete. So $X$ is a Hilbert space.

For $u \in X$, we denote

$$
\|u\|_{\infty}=\max _{j \in\{0,1, \ldots, p\}}\left\{\max _{t \in\left[t_{j}, t_{j+1}\right]}\left|u^{j}(t)\right|\right\},
$$

then we can get the following result.

Lemma 2.2. Let $c=\max _{j \in\{0,1, \ldots, p\}}\left\{\left(t_{j+1}-t_{j}\right)^{-1 / 2},\left(t_{j+1}-t_{j}\right)^{1 / 2}\right\}$, then

$$
\|u\|_{\infty} \leq c\|u\|
$$

for every $u \in X$.

Proof. Let $u \in X$, by mean value theorem, there exists $\tau \in\left[t_{j}, t_{j+1}\right]$, such that

$$
u^{j}(\tau)=\frac{1}{t_{j+1}-t_{j}} \int_{t_{j}}^{t_{j+1}} u^{j}(\theta) d \theta
$$


For $t \in\left[t_{j}, t_{j+1}\right]$, from Hölder inequality, we have

$$
\begin{aligned}
\left|u^{j}(t)\right| & =\left|u^{j}(\tau)+\int_{\tau}^{t}\left(u^{j}\right)^{\prime}(\theta) d \theta\right| \\
& \leq \frac{1}{t_{j+1}-t_{j}} \int_{t_{j}}^{t_{j+1}}\left|u^{j}(\theta)\right| d \theta+\int_{t_{j}}^{t_{j+1}}\left|\left(u^{j}\right)^{\prime}(\theta)\right| d \theta \\
& \leq\left(t_{j+1}-t_{j}\right)^{-1 / 2}\left(\int_{t_{j}}^{t_{j+1}}\left|u^{j}(\theta)\right|^{2} d \theta\right)^{1 / 2}+\left(t_{j+1}-t_{j}\right)^{1 / 2}\left(\int_{t_{j}}^{t_{j+1}}\left|\left(u^{j}\right)^{\prime}(\theta)\right|^{2} d \theta\right)^{1 / 2} \\
& \leq \max _{j \in\{0,1, \ldots, p\}}\left\{\left(t_{j+1}-t_{j}\right)^{-1 / 2},\left(t_{j+1}-t_{j}\right)^{1 / 2}\right\}\left(\int_{t_{j}}^{t_{j+1}}\left(\left|u^{j}(\theta)\right|^{2}+\left|\left(u^{j}\right)^{\prime}(\theta)\right|^{2}\right) d \theta\right)^{1 / 2} \\
& =c\left\|u^{j}\right\|_{H^{1}\left(t_{j}, t_{j+1}\right)} \leq c\|u\| .
\end{aligned}
$$

Definition 2.3. A function $u \in L^{2}(0, T)$ is said to be a classical solution of problem $(P)$, if $u_{\mid\left(t_{j}, t_{j+1}\right)} \in C^{2}\left(t_{j}, t_{j+1}\right)$ for $j=0,1,2, \ldots, p$, and $u$ satisfies $(1.9)$ on $[0, T] \backslash\left\{t_{1}, t_{2}, \ldots, t_{p}\right\}$, the limits $u^{\prime}\left(t_{j}^{+}\right), u^{\prime}\left(t_{j}^{-}\right), u\left(t_{j}^{+}\right), u\left(t_{j}^{-}\right)$exist, and (1.10), (1.11), and (1.12) hold.

For convenience, denote

$$
\alpha_{1}=\left\{\begin{array}{l}
\frac{\alpha}{\beta}, \quad \beta \neq 0, \\
0, \quad \beta=0,
\end{array} \quad \alpha_{2}=\left\{\begin{array}{ll}
\frac{\gamma}{\sigma}, & \sigma \neq 0, \\
0, & \sigma=0,
\end{array} \quad F(t, u)=\int_{0}^{u} f(t, \xi) d \xi .\right.\right.
$$

For $u \in X$, set

$$
\begin{aligned}
\varphi(u)= & \frac{1}{2} \int_{0}^{T}\left[\left(u^{\prime}(t)\right)^{2}+u^{2}(t)\right] d t+\frac{1}{2} \alpha_{1} u^{2}(0)+\frac{1}{2} \alpha_{2} u^{2}(T)-\int_{0}^{T} F(t, u(t)) d t \\
& -\sum_{j=1}^{p} \int_{0}^{u\left(t_{j}^{-}\right)} I_{j}(s) d s .
\end{aligned}
$$

From the assumption of the continuity of $f$ and $I_{j}(j=1,2, \ldots, p)$, we can see that $\varphi \in C^{1}(X, \mathbb{R})$ and

$$
\begin{aligned}
\left(\varphi^{\prime}(u), v\right)= & \int_{0}^{T}\left[u^{\prime}(t) v^{\prime}(t)+u(t) v(t)\right] d t+\alpha_{1} u(0) v(0)+\alpha_{2} u(T) v(T) \\
& -\int_{0}^{T} f(t, u(t)) v(t) d t-\sum_{j=1}^{p} I_{j}\left(u\left(t_{j}^{-}\right)\right) v\left(t_{j}^{-}\right)
\end{aligned}
$$

for $u, v \in X$. 
Lemma 2.4. If the function $u \in X$ is a critical point of $\varphi$, then $u$ is a classical solution of problem $(P)$.

Proof. Suppose that $u \in X$ is a critical point of $\varphi$, then $\left(\varphi^{\prime}(u), v\right)=0$ for every $v \in X$. By (2.16), we have

$$
\int_{0}^{T}\left[u^{\prime} v^{\prime}+u v\right] d t+\alpha_{1} u(0) v(0)+\alpha_{2} u(T) v(T)-\int_{0}^{T} f(t, u) v d t-\sum_{j=1}^{p} I_{j}\left(u\left(t_{j}^{-}\right)\right) v\left(t_{j}^{-}\right)=0 .
$$

For $j \in\{0,1,2, \ldots, p\}$, choose $v=0$ on $[0, T] \backslash\left(t_{j}, t_{j+1}\right)$ and $v_{\mid\left(t_{j}, t_{j+1}\right)} \in C_{0}^{\infty}\left(t_{j}, t_{j+1}\right)$, then $v \in X$ and

$$
\int_{t_{j}}^{t_{j+1}} u^{\prime} v^{\prime} d t=\int_{t_{j}}^{t_{j+1}}(f(t, u)-u) v d t
$$

By the definition of $H^{1}\left(t_{j}, t_{j+1}\right)$ and since $u_{\left.\right|_{\left(t_{j}, t_{j+1}\right)}} \in H^{1}\left(t_{j}, t_{j+1}\right) \subset C\left[t_{j}, t_{j+1}\right], f \in$

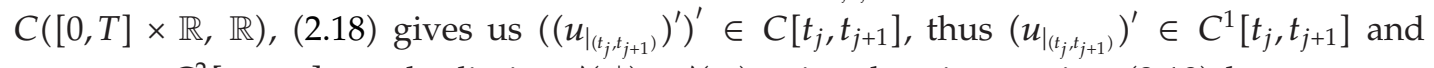
$u_{\left(t_{j}, t_{j+1}\right)} \in C^{2}\left[t_{j}, t_{j+1}\right]$, so the limits $u^{\prime}\left(t_{j}^{+}\right), u^{\prime}\left(t_{j}^{-}\right)$exist, then integrating (2.18) by parts, we obtain

$$
\int_{t_{j}}^{t_{j+1}}\left(-u^{\prime \prime}+u-f(t, u)\right) v d t=0, \quad v \in X, v_{\mid\left(t_{j}, t_{j+1}\right)} \in C_{0}^{\infty}\left(t_{j}, t_{j+1}\right) .
$$

So

$$
-u^{\prime \prime}+u-f(t, u)=0, \quad t \in\left(t_{j}, t_{j+1}\right) .
$$

Thus, we can get that $u$ satisfies (1.9) for $t \in(0, T) \backslash\left\{t_{1}, \ldots, t_{p}\right\}$.

In view of (2.17), (2.19), and $-\Delta u\left(t_{j}\right)=A_{j} u\left(t_{j}^{-}\right)$, we get that

$$
\begin{aligned}
0= & \sum_{j=0}^{p} \int_{t_{j}}^{t_{j+1}} u^{\prime} v^{\prime} d t+\int_{0}^{T}(u-f(t, u)) v d t+\alpha_{1} u(0) v(0)+\alpha_{2} u(T) v(T)-\sum_{j=1}^{p} I_{j}\left(u\left(t_{j}^{-}\right)\right) v\left(t_{j}^{-}\right) \\
= & \sum_{j=0}^{p}\left(u^{\prime}\left(t_{j+1}^{-}\right) v\left(t_{j+1}^{-}\right)-u^{\prime}\left(t_{j}^{+}\right) v\left(t_{j}^{+}\right)\right)+\int_{0}^{T}\left(-u^{\prime \prime}+u-f(t, u)\right) v d t+\alpha_{1} u(0) v(0) \\
& +\alpha_{2} u(T) v(T)-\sum_{j=1}^{p} I_{j}\left(u\left(t_{j}^{-}\right)\right) v\left(t_{j}^{-}\right)
\end{aligned}
$$




$$
\begin{aligned}
= & \sum_{j=1}^{p}\left(u^{\prime}\left(t_{j}^{-}\right) v\left(t_{j}^{-}\right)-u^{\prime}\left(t_{j}^{+}\right) v\left(t_{j}^{-}\right)+u^{\prime}\left(t_{j}^{+}\right) v\left(t_{j}^{-}\right)-u^{\prime}\left(t_{j}^{+}\right) v\left(t_{j}^{+}\right)\right)+u^{\prime}(T) v(T)-u^{\prime}(0) v(0) \\
& +\alpha_{1} u(0) v(0)+\alpha_{2} u(T) v(T)-\sum_{j=1}^{p} I_{j}\left(u\left(t_{j}^{-}\right)\right) v\left(t_{j}^{-}\right) \\
= & -\sum_{j=1}^{p}\left(\Delta u^{\prime}\left(t_{j}\right)+I_{j}\left(u\left(t_{j}^{-}\right)\right)-A_{j} u^{\prime}\left(t_{j}^{+}\right)\right) v\left(t_{j}^{-}\right) \\
& +\left(\alpha_{1} u(0)-u^{\prime}(0)\right) v(0)+\left(\alpha_{2} u(T)+u^{\prime}(T)\right) v(T) .
\end{aligned}
$$

If we choose $v \in X$, such that $v(0)=v(T)=0$, then it follows from the above equality that

$$
-\Delta u^{\prime}\left(t_{j}\right)=I_{j}\left(u\left(t_{j}^{-}\right)\right)-A_{j} u^{\prime}\left(t_{j}^{+}\right)
$$

and the impulsive condition (1.11) holds.

If $\beta=\sigma=0$, then from the definition of $X$, we know that $u(0)=u(T)=0$, and the boundary condition (1.12) holds. If $\beta \neq 0, \sigma \neq 0$, in view of (2.21) and (2.22), we can conclude that

$$
\left(\alpha_{1} u(0)-u^{\prime}(0)\right) v(0)+\left(\alpha_{2} u(T)+u^{\prime}(T)\right) v(T)=0, \quad v \in X
$$

Since $v(0)$ and $v(T)$ are arbitrary, we deduce that

$$
\alpha_{1} u(0)-u^{\prime}(0)=0, \quad \alpha_{2} u(T)+u^{\prime}(T)=0,
$$

and $u$ satisfies the boundary condition (1.12). If one of $\beta$ and $\sigma$ is equal to 0 , similarly we can obtain the conclusion.

Therefore, $u$ is a classical solution of problem $(P)$.

Lemma 2.5 (Mountain Pass theorem [10, Theorem 2.2]). Let E be a real Banach space and $\varphi \in$ $C^{1}(E, \mathbb{R})$ satisfying the $(\mathrm{PS})$ condition. Suppose $\varphi(0)=0$ and

$\left(C_{1}\right)$ there are constants $\rho, \alpha>0$ such that $\left.\varphi\right|_{\partial B_{\rho}} \geq \alpha$, where $B_{\rho}=\{x \in E:\|x\|<\rho\}$;

$\left(C_{2}\right)$ there is an $e \in E \backslash \overline{B_{\rho}}$ such that $\varphi(e) \leq 0$.

Then $\varphi$ possesses a critical value $c \geq \alpha$. Moreover $c$ can be characterized as

$$
c=\inf _{g \in \Gamma} \max _{u \in g([0,1])} \varphi(u)
$$

where

$$
\Gamma=\{g \in C([0,1], E) \mid g(0)=0, g(1)=e\} .
$$


Lemma 2.6 (see [10, Theorem 9.12]). Let $E$ be an infinite dimensional Banach space, and let $\varphi \in$ $C^{1}(E, \mathbb{R})$ be even functional which satisfies the (PS) condition, and $\varphi(0)=0$. If $E=V \oplus X$, where $V$ is finite dimensional, and $\varphi$ satisfies

$\left(C_{1}^{\prime}\right)$ there are constants $\rho, \alpha>0$ such that $\left.\varphi\right|_{\partial B_{\rho} \cap \mathrm{X}} \geq \alpha$, where $B_{\rho}=\{x \in E:\|x\|<\rho\}$;

$\left(C_{2}^{\prime}\right)$ for each finite dimensional subspace $\widetilde{E} \subset E$, there is an $R=R(\widetilde{E})$ such that $\varphi \leq 0$ on $\tilde{E} \backslash B_{R(\tilde{E})}$.

Then $\varphi$ possesses an unbounded sequence of critical values.

\section{Main Results}

Lemma 3.1. If there exist $\mu>2$ and $\delta>0$ such that

$$
\begin{gathered}
0<\mu F(t, x) \leq x f(t, x), \quad \text { for } t \in[0, T],|x| \geq \delta, \\
0<\mu \int_{0}^{x} I_{j}(s) d s \leq x I_{j}(x), \quad \text { for }|x| \geq \delta, j=1,2, \ldots, p,
\end{gathered}
$$

then the functional $\varphi$ satisfies the (PS) condition.

Proof. Let $\left\{u_{n}\right\}$ be a sequence in $X$ satisfying that $\left\{\varphi\left(u_{n}\right)\right\}$ is bounded and $\varphi^{\prime}\left(u_{n}\right) \rightarrow 0$ as $n \rightarrow \infty$. Now we shall prove that $\left\{u_{n}\right\}$ is a bounded sequence in X. By (2.15), (2.16), (3.1), and (3.2), we have

$$
\begin{aligned}
& \mu \varphi\left(u_{n}\right)-\left(\varphi^{\prime}\left(u_{n}\right), u_{n}\right) \\
&=\frac{\mu}{2} \int_{0}^{T}\left[\left(u_{n}^{\prime}\right)^{2}+\left(u_{n}\right)^{2}\right] d t+\frac{\mu \alpha_{1}}{2}\left(u_{n}(0)\right)^{2}+\frac{\mu \alpha_{2}}{2}\left(u_{n}(T)\right)^{2} \\
& \quad-\mu \int_{0}^{T} F\left(t, u_{n}\right) d t-\mu \sum_{j=1}^{p} \int_{0}^{u_{n}\left(t_{j}^{-}\right)} I_{j}(s) d s \\
& \quad-\int_{0}^{T}\left[\left(u_{n}^{\prime}\right)^{2}+\left(u_{n}\right)^{2}\right] d t-\alpha_{1}\left(u_{n}(0)\right)^{2}-\alpha_{2}\left(u_{n}(T)\right)^{2} \\
& \quad+\int_{0}^{T} f\left(t, u_{n}\right) u_{n} d t+\sum_{j=1}^{p} I_{j}\left(u_{n}\left(t_{j}^{-}\right)\right) u_{n}\left(t_{j}^{-}\right) \\
& \geq\left(\frac{\mu}{2}-1\right)\left(\left\|u_{n}\right\|^{2}+\alpha_{1}\left(u_{n}(0)\right)^{2}+\alpha_{2}\left(u_{n}(T)\right)^{2}\right)+T \min _{t \in[0, T],|x| \leq \delta}(f(t, x) x-\mu F(t, x)) \\
&+\sum_{j=1}^{p} \min \left(I_{j}(x) x-\mu \int_{0}^{x} I_{j}(s) d s\right) \\
& \geq\left(\frac{\mu}{2}-1\right)\left\|u_{n}\right\|^{2}+M,
\end{aligned}
$$


where $M=T \min _{t \in[0, T],|x| \leq \delta}(f(t, x) x-\mu F(t, x))+\sum_{j=1}^{p} \min _{|x| \leq \delta}\left(I_{j}(x) x-\mu \int_{0}^{x} I_{j}(s) d s\right)$ is a constant. So $\left\{u_{n}\right\}$ is bounded in $X$.

From the reflexivity of $X$, we may extract a weakly convergent subsequence that, for simplicity, we call $\left\{u_{n}\right\}, u_{n} \rightarrow u$. In what follows, we will prove that $\left\{u_{n}\right\}$ strongly converges to $u$. By $u_{n} \rightarrow u$, we see that, for $j=0,1, \ldots, p, u_{n}^{j} \rightarrow u^{j}$ in $H^{1}\left(t_{j}, t_{j+1}\right)$, and $\left\{u_{n}^{j}\right\}$ converges to $u^{j}$ in $C\left[t_{j}, t_{j+1}\right]$. From the continuity of $f$ and $I_{j}(j=1,2, \ldots, p)$, we know that

$$
\begin{gathered}
\int_{0}^{T}\left(f\left(t, u_{n}\right)-f(t, u)\right)\left(u_{n}-u\right) d t \longrightarrow 0, \quad \text { as } n \longrightarrow \infty, \\
\sum_{j=1}^{p}\left(I_{j}\left(u_{n}\left(t_{j}^{-}\right)\right)-I_{j}\left(u\left(t_{j}^{-}\right)\right)\right)\left(u_{n}\left(t_{j}^{-}\right)-u\left(t_{j}^{-}\right)\right) \longrightarrow 0, \quad \text { as } n \longrightarrow \infty .
\end{gathered}
$$

In view of (2.16), we get

$$
\begin{aligned}
& \left(\varphi^{\prime}\left(u_{n}\right)-\varphi^{\prime}(u), u_{n}-u\right) \\
& \quad=\left\|u_{n}-u\right\|^{2}+\alpha_{1}\left(u_{n}(0)-u(0)\right)^{2}+\alpha_{2}\left(u_{n}(T)-u(T)\right)^{2} \\
& \quad+\int_{0}^{T}\left(f\left(t, u_{n}\right)-f(t, u)\right)\left(u_{n}-u\right) d t+\sum_{j=1}^{p}\left(I_{j}\left(u_{n}\left(t_{j}^{-}\right)\right)-I_{j}\left(u\left(t_{j}^{-}\right)\right)\right)\left(u_{n}\left(t_{j}^{-}\right)-u\left(t_{j}^{-}\right)\right) .
\end{aligned}
$$

By $\varphi^{\prime}\left(u_{n}\right) \rightarrow 0$ and $u_{n} \rightarrow u$, we obtain that

$$
\left(\varphi^{\prime}\left(u_{n}\right)-\varphi^{\prime}(u), u_{n}-u\right) \longrightarrow 0, \quad \text { as } n \longrightarrow \infty
$$

From the previous discussions, it is easy to see that $\left\|u_{n}-u\right\| \rightarrow 0$ as $n \rightarrow \infty$. Therefore, $\varphi$ satisfies the (PS) condition.

Theorem 3.2. Suppose that (3.1), (3.2) hold and

$$
\begin{gathered}
f(t, x)=o(x), \quad x \longrightarrow 0, t \in[0, T], \\
I_{j}(x)=o(x), \quad x \longrightarrow 0, j=1,2, \ldots, p,
\end{gathered}
$$

then problem $(P)$ has at least one nontrivial solution.

Proof. In order to show that problem $(P)$ has at least one nontrivial solution, that is, $\varphi$ has at least one nontrivial critical point, it is sufficient to check the conditions in Lemma 2.5. Clearly $\varphi \in C^{1}(X, R), \varphi(0)=0$, and $\varphi$ satisfies the (PS) condition from Lemma 3.1.

First, it follows from (3.7) and (3.8) that there exists a constant $\varepsilon>0$, such that for $|x| \leq \varepsilon$ :

$$
|F(t, x)| \leq \frac{1}{8 c^{2} T} x^{2}, \quad \int_{0}^{x} I_{j}(s) d s \leq \frac{1}{8 c^{2} p} x^{2}, \quad j=1,2, \ldots, p,
$$


where $c$ is given in Lemma 2.2. Choose $u \in X$ with $\|u\| \leq \varepsilon / c$, then $\|u\|_{\infty} \leq \varepsilon$, and by (3.9) and (2.11), we have

$$
\begin{aligned}
\varphi(u) & =\frac{1}{2}\|u\|^{2}+\frac{1}{2} \alpha_{1} u^{2}(0)+\frac{1}{2} \alpha_{2} u^{2}(T)-\int_{0}^{T} F(t, u(t)) d t-\sum_{j=1}^{p} \int_{0}^{u\left(t_{j}^{-}\right)} I_{j}(s) d s \\
& \geq \frac{1}{2}\|u\|^{2}-\int_{0}^{T} \frac{1}{8 c^{2} T} u^{2}(t) d t-\sum_{j=1}^{p} \frac{1}{8 c^{2} p}\left(u\left(t_{j}^{-}\right)\right)^{2} \\
& \geq \frac{1}{2}\|u\|^{2}-\int_{0}^{T} \frac{1}{8 c^{2} T}\|u\|_{\infty}^{2} d t-\sum_{j=1}^{p} \frac{1}{8 c^{2} p}\|u\|_{\infty}^{2} \\
& =\frac{1}{2}\|u\|^{2}-\frac{1}{4 c^{2}}\|u\|_{\infty}^{2} \geq \frac{1}{2}\|u\|^{2}-\frac{1}{4}\|u\|^{2}=\frac{1}{4}\|u\|^{2} .
\end{aligned}
$$

If we choose $\alpha=\varepsilon^{2} / 4 c^{2}, \rho=\varepsilon / c$, then $\left.\varphi\right|_{\partial B_{\rho}} \geq \alpha$. Thus, condition $\left(C_{1}\right)$ of Lemma 2.5 holds.

By (3.1) and (3.2), we know that there are positive constants $c_{1}, c_{2}, d_{1}$, and $d_{2}$ such that

$$
\begin{gathered}
F(t, x) \geq c_{1}|x|^{\mu}-c_{2}, \quad(t, x) \in[0, T] \times \mathbb{R}, \\
\int_{0}^{x} I_{j}(s) d s \geq d_{1}|x|^{\mu}-d_{2}, \quad x \in \mathbb{R}, j=1,2, \ldots, p .
\end{gathered}
$$

If we choose $u_{0} \in X \backslash\{0\}$, then for any $r>0$, we have

$$
\begin{aligned}
& \varphi\left(r u_{0}\right) \\
& =\frac{1}{2}\left\|r u_{0}\right\|^{2}+\frac{1}{2} \alpha_{1}\left(r u_{0}(0)\right)^{2}+\frac{1}{2} \alpha_{2}\left(r u_{0}(T)\right)^{2}-\int_{0}^{T} F\left(t, r u_{0}\right) d t-\sum_{j=1}^{p} \int_{0}^{r u_{0}\left(t_{j}^{-}\right)} I_{j}(s) d s \\
& \leq \frac{1}{2}\left\|r u_{0}\right\|^{2}+\frac{r^{2}}{2}\left(\alpha_{1}\left(u_{0}(0)\right)^{2}+\alpha_{2}\left(u_{0}(T)\right)^{2}\right)-\int_{0}^{T}\left(c_{1}\left|r u_{0}\right|^{\mu}-c_{2}\right) d t-\sum_{j=1}^{p} d_{1}\left|r u_{0}\left(t_{j}^{-}\right)\right|^{\mu}+p d_{2} \\
& =\frac{r^{2}}{2}\left(\left\|u_{0}\right\|^{2}+\alpha_{1}\left(u_{0}(0)\right)^{2}+\alpha_{2}\left(u_{0}(T)\right)^{2}\right)-\left(c_{1} \int_{0}^{T}\left|u_{0}\right|^{\mu} d t+d_{1} \sum_{j=1}^{p}\left|u_{0}\left(t_{j}^{-}\right)\right|^{\mu}\right) r^{\mu}+c_{2} T+p d_{2} .
\end{aligned}
$$

Since $\mu>2$, we can find $r>1$ such that $\left\|r u_{0}\right\|>\rho$ and $\varphi\left(r u_{0}\right)<0$. Hence the condition $\left(C_{2}\right)$ of Lemma 2.5 is satisfied.

Theorem 3.3. Under the same assumptions as Theorem 3.2, if $f(t, x)$ is odd in $x$, and $I_{j}(j=$ $1,2, \ldots, p)$ are odd, then problem $(P)$ has infinitely many solutions.

Proof. The proof follows the analogous ideas as that we have developed for Theorem 3.2. By the assumption we know that $\varphi(u)$ is even, $\varphi \in C^{1}(X, R), \varphi(0)=0$, and $\varphi$ satisfies the (PS) condition from Lemma 3.1. 
If we let $V$ be any finite dimensional subspace of $X$, and $W=V^{\perp}$, then for any $u \in W$ with $\|u\| \leq \varepsilon / c$, it follows from (3.10) that

$$
\varphi(u) \geq \alpha, \quad \text { for } u \in \partial B_{\rho} \cap W,
$$

where $\alpha=\varepsilon^{2} / 4 c^{2}, \rho=\varepsilon / c$. So $\varphi$ satisfies the condition $\left(C_{1}^{\prime}\right)$. On the other hand, fix $u_{0} \in$ $V \backslash\{0\}$, the same as (3.13), we can find some $r>1$ such that

$$
\left\|r u_{0}\right\|>\rho, \quad \varphi\left(r u_{0}\right)<0,
$$

then $\left(C_{2}^{\prime}\right)$ of Lemma 2.6 is true. Thus, $\varphi$ possesses an infinite number of critical points, that is, problem $(P)$ has infinitely many solutions.

When $A_{j} \equiv 0$ and $I_{j} \equiv 0$ for $j=1,2, \ldots, p$, problem $(P)$ degenerates to the following problem $\left(P_{0}\right)$ :

$$
\begin{gathered}
-u^{\prime \prime}(t)+u(t)=f(t, u(t)), \quad t \in[0, T], \\
\alpha u(0)-\beta u^{\prime}(0)=0, \quad \gamma u(T)+\sigma u^{\prime}(T)=0 .
\end{gathered}
$$

Now, we show the nonexistence of solutions for problem $\left(P_{0}\right)$.

Lemma 3.4. Assume that $f$ satisfies

$$
x f(t, x) \leq 0, \quad(t, x) \in[0, T] \times \mathbb{R} .
$$

Then, $\left(P_{0}\right)$ has no nontrivial solution.

Proof. If $u$ is a nontrivial solution of $\left(P_{0}\right)$, then we have

$$
\begin{aligned}
0 & =-\int_{0}^{T} u^{\prime \prime} u d t+\int_{0}^{T} u^{2} d t-\int_{0}^{T} f(t, u) u d t \\
& =\int_{0}^{T}\left[\left(u^{\prime}\right)^{2}+u^{2}\right] d t+\alpha_{1} u^{2}(0)+\alpha_{2} u^{2}(T)-\int_{0}^{T} f(t, u) u d t \\
& =\|u\|^{2}+\alpha_{1} u^{2}(0)+\alpha_{2} u^{2}(T)-\int_{0}^{T} f(t, u) u d t \geq 0 .
\end{aligned}
$$

Since $\alpha_{1} \geq 0, \alpha_{2} \geq 0$ and (3.17), we obtain $u \equiv 0$.

Remark 3.5. By Lemma 3.4, if we get a solution of problem $(P)$ under the assumption of (3.17), then the solution is generated by impulses.

The next theorem gives some sufficient conditions that problem $(P)$ has at least one solution and infinitely many solutions generated by impulses under the assumption of (3.17), compared with Theorem 3.2, we change the conditions on nonlinearity and make certain requirements about $A_{j}, j=1,2, \ldots, p$. 
Theorem 3.6. Suppose $0 \leq A_{j} \leq 1$ for $j=1,2, \ldots, p,(3.2),(3.8)$, and (3.17) hold, and there exist $a>0$ and $\eta \in(0,1)$ such that

$$
f(t, x) \geq-a|x|^{\eta}, \quad(t, x) \in[0, T] \times \mathbb{R} .
$$

Then problem $(P)$ has at least one nontrivial solution generated by impulses. Furthermore, if $f(t, x)$ is odd in $x$, and $I_{j}(j=1,2, \ldots, p)$ are odd, then problem $(P)$ has infinitely many solutions generated by impulses.

Proof. Firstly, we show that $\varphi$ satisfies the (PS) condition. Let $\left\{u_{n}\right\}$ be a sequence in $X$ satisfying that $\left\{\varphi\left(u_{n}\right)\right\}$ is bounded and $\varphi^{\prime}\left(u_{n}\right) \rightarrow 0$ as $n \rightarrow \infty$. Now, we shall prove that $\left\{u_{n}\right\}$ is a bounded sequence in $X$.

Equation (3.17) implies that

$$
F(t, x) \leq 0, \quad(t, x) \in[0, T] \times \mathbb{R}
$$

By (3.2), (3.19), and (3.20), we have

$$
\begin{aligned}
\mu \varphi\left(u_{n}\right)-\left(\varphi^{\prime}\left(u_{n}\right), u_{n}\right) \\
=\left(\frac{\mu}{2}-1\right) \int_{0}^{T}\left[\left(u_{n}^{\prime}\right)^{2}+\left(u_{n}\right)^{2}\right] d t+\left(\frac{\mu}{2}-1\right) \alpha_{1}\left(u_{n}(0)\right)^{2}+\left(\frac{\mu}{2}-1\right) \alpha_{2}\left(u_{n}(T)\right)^{2} \\
\quad-\mu \int_{0}^{T} F\left(t, u_{n}\right) d t+\int_{0}^{T} f\left(t, u_{n}\right) u_{n} d t-\mu \sum_{j=1}^{p} \int_{0}^{u_{n}\left(t_{j}^{-}\right)} I_{j}(s) d s+\sum_{j=1}^{p} I_{j}\left(u_{n}\left(t_{j}^{-}\right)\right) u_{n}\left(t_{j}^{-}\right) \\
\geq\left(\frac{\mu}{2}-1\right)\left\|u_{n}\right\|^{2}+\int_{0}^{T} f\left(t, u_{n}\right) u_{n} d t-\mu \sum_{j=1}^{p} \int_{0}^{u_{n}\left(t_{j}^{-}\right)} I_{j}(s) d s+\sum_{j=1}^{p} I_{j}\left(u_{n}\left(t_{j}^{-}\right)\right) u_{n}\left(t_{j}^{-}\right) \\
\geq\left(\frac{\mu}{2}-1\right)\left\|u_{n}\right\|^{2}-a T\left\|u_{n}\right\|_{\infty}^{\eta+1}+\sum_{j=1}^{p} \min _{|x| \leq \delta}\left(I_{j}(x) x-\mu \int_{0}^{x} I_{j}(s) d s\right) \\
\geq\left(\frac{\mu}{2}-1\right)\left\|u_{n}\right\|^{2}-a T c^{\eta+1}\left\|u_{n}\right\|^{\eta+1}+M_{1},
\end{aligned}
$$

where $M_{1}=\sum_{j=1}^{p} \min _{|x| \leq \delta}\left(I_{j}(x) x-\mu \int_{0}^{x} I_{j}(s) d s\right)$ is a constant. So $\left\{u_{n}\right\}$ is bounded in $X$, since $\eta<1, \mu>2$. Similar to the proof in Lemma 3.1, we can see that $\left\{u_{n}\right\}$ has a convergent subsequence in $X$. So, $\varphi$ satisfies the (PS) condition.

From (3.8), there exists a constant $\varepsilon_{1}>0$ such that for $j=1,2, \ldots, p$, we have

$$
\int_{0}^{x} I_{j}(s) d s \leq \frac{1}{4 c^{2} p} x^{2}, \quad|x| \leq \varepsilon_{1}
$$


where $c$ is given in Lemma 2.2. Hence combining this with (3.20), for $u \in X$ with $\|u\| \leq \varepsilon_{1} / c$, we have

$$
\begin{aligned}
\varphi(u) & =\frac{1}{2}\|u\|^{2}+\frac{1}{2} \alpha_{1} u^{2}(0)+\frac{1}{2} \alpha_{2} u^{2}(T)-\int_{0}^{T} F(t, u(t)) d t-\sum_{j=1}^{p} \int_{0}^{u\left(t_{j}^{-}\right)} I_{j}(s) d s \\
& \geq \frac{1}{2}\|u\|^{2}-\sum_{j=1}^{p} \int_{0}^{u\left(t_{j}^{-}\right)} I_{j}(s) d s \\
& \geq \frac{1}{2}\|u\|^{2}-\sum_{j=1}^{p} \frac{1}{4 c^{2} p} u^{2}\left(t_{j}^{-}\right) \\
& \geq \frac{1}{2}\|u\|^{2}-\frac{1}{4 c^{2}}\|u\|_{\infty}^{2} \\
& \geq \frac{1}{2}\|u\|^{2}-\frac{1}{4}\|u\|^{2}=\frac{1}{4}\|u\|^{2} .
\end{aligned}
$$

If we choose $\alpha=\varepsilon_{1}^{2} / 4 c^{2}, \rho=\varepsilon_{1} / c$, then $\left.\varphi\right|_{\partial B_{\rho}} \geq \alpha$. Thus, condition $\left(C_{1}\right)$ in Lemma 2.5 holds. For some constant $k>0$, let

$$
\bar{u}(t)= \begin{cases}k t, & t \in\left[0, t_{1}\right), \\ k t_{1} \prod_{i=1}^{j}\left(1-A_{i}\right), & t \in\left(t_{j}, t_{j+1}\right), j=1, \ldots, p-1, \\ \frac{k t_{1} \prod_{i=1}^{p}\left(1-A_{i}\right)}{t_{p}-T}(t-T), & t \in\left(t_{p}, T\right] .\end{cases}
$$

Clearly, $\bar{u} \in X$ and $\bar{u}(t) \geq 0$ for $t \in[0, T]$. Then for any $r>0$, by (3.19), we have

$$
F(t, r \bar{u}(t))=\int_{0}^{r \bar{u}(t)} f(t, s) d s \geq-\int_{0}^{r \bar{u}(t)} a|s|^{\eta} d s=-\frac{a}{\eta+1}|r \bar{u}(t)|^{\eta+1} \geq-\frac{a}{\eta+1}\|r \bar{u}\|_{\infty}^{\eta+1} .
$$

In view of (3.25) and (3.12), one can get

$$
\begin{aligned}
\varphi(r \bar{u}) & =\frac{1}{2}\|r \bar{u}\|^{2}+\frac{1}{2} \alpha_{1}(r \bar{u}(0))^{2}+\frac{1}{2} \alpha_{2}(r \bar{u}(T))^{2}-\int_{0}^{T} F(t, r \bar{u}) d t-\sum_{j=1}^{p} \int_{0}^{r \bar{u}\left(t_{j}^{-}\right)} I_{j}(s) d s \\
& \leq \frac{r^{2}}{2}\left(\|\bar{u}\|^{2}+\alpha_{1}(\bar{u}(0))^{2}+\alpha_{2}(\bar{u}(T))^{2}\right)+\frac{a T c^{\eta+1}}{\eta+1}\|\bar{u}\|^{\eta+1} r^{\eta+1}-\sum_{j=1}^{p} d_{1}\left|\bar{u}\left(t_{j}^{-}\right)\right|^{\mu} r^{\mu}+p d_{2} .
\end{aligned}
$$

Since $\mu>2, \eta<1$, we can find $r>1$ such that $\|r \bar{u}\|>\rho$ and $\varphi(r \bar{u})<0$. Hence, the condition $\left(C_{2}\right)$ in Lemma 2.5 is satisfied. 
Finally, by Remark 3.5 and Lemma 2.5, the problem $(P)$ has at least one nontrivial solution generated by impulses. Analogous to the proof of Theorem 3.3, we know that when $f(t, x)$ is odd about $x$, and $I_{j}(j=1,2, \ldots, p)$ are odd, problem $(P)$ has an infinite number of solutions which are generated by impulses.

Theorem 3.7. Suppose (3.17) holds, $0<A_{j}<1$ for $j=1,2, \ldots, p$, and there exist positive constants $a_{0}, a_{j}, \tilde{a}_{j}, b_{j}$ and $\eta_{0}, \eta_{j}, v_{j}$ which satisfy $\eta_{j} \in(0,1)$ and $0<v_{j}<\min \left\{\eta_{0}, 1\right\}$ such that

(i) $I_{j}(x) \leq a_{j}|x|^{\eta_{j}}+b_{j}$ for $x \in \mathbb{R}, j=1,2, \ldots, p$;

(ii) $I_{j}(x) \geq \tilde{a}_{j}|x|^{v_{j}}$ for $|x| \leq 1, x \neq 0$, and $f(t, x) \geq-a_{0}|x|^{\eta_{0}}$ for $|x| \leq 1, t \in[0, T]$.

Then problem $(P)$ has at least one nontrivial solution generated by impulses.

Proof. Firstly, we show that $\varphi$ is weakly lower semi continuous. Let $\left\{u_{n}\right\}$ be a sequence which weakly converges to some $u$ in $X$, then

$$
\|u\| \leq \liminf _{n \rightarrow \infty}\left\|u_{n}\right\|
$$

On the other hand, we have that $\left\{\left.u_{n}\right|_{\left(t_{j}, t_{j+1}\right)}\right\}$ weakly converges to $\left.u\right|_{\left(t_{j}, t_{j+1}\right)}$ in $H^{1}\left(t_{j}, t_{j+1}\right)$, then $\left\{\left.u_{n}\right|_{\left(t_{j}, t_{j+1}\right)}\right\}$ uniformly converges to $\left.u\right|_{\left(t_{j}, t_{j+1}\right)}$ on $\left[t_{j}, t_{j+1}\right]$, then

$$
\begin{aligned}
& \lim _{n \rightarrow \infty}\left(\frac{1}{2} \alpha_{1} u_{n}^{2}(0)+\frac{1}{2} \alpha_{2} u_{n}^{2}(T)-\int_{0}^{T} F\left(t, u_{n}(t)\right) d t-\sum_{j=1}^{p} \int_{0}^{u_{n}\left(t_{j}^{-}\right)} I_{j}(s) d s\right) \\
& \quad=\lim _{n \rightarrow \infty}\left(\frac{1}{2} \alpha_{1} u_{n}^{2}(0)+\frac{1}{2} \alpha_{2} u_{n}^{2}(T)-\sum_{j=0}^{p} \int_{t_{j}}^{t_{j+1}} F\left(t, u_{n}(t)\right) d t-\sum_{j=1}^{p} \int_{0}^{u_{n}\left(t_{j}^{-}\right)} I_{j}(s) d s\right) \\
& \quad=\frac{1}{2} \alpha_{1} u^{2}(0)+\frac{1}{2} \alpha_{2} u^{2}(T)-\sum_{j=0}^{p} \int_{t_{j}}^{t_{j+1}} F(t, u(t)) d t-\sum_{j=1}^{p} \int_{0}^{u\left(t_{j}^{-}\right)} I_{j}(s) d s \\
& \quad=\frac{1}{2} \alpha_{1} u^{2}(0)+\frac{1}{2} \alpha_{2} u^{2}(T)-\int_{0}^{T} F(t, u(t)) d t-\sum_{j=1}^{p} \int_{0}^{u\left(t_{j}^{-}\right)} I_{j}(s) d s .
\end{aligned}
$$

From (3.27) and (3.28), we conclude that $\varphi(u) \leq \liminf _{n \rightarrow \infty} \varphi\left(u_{n}\right)$.

Next, we verify that $\varphi$ is coercive. 
From (3.17), the hypothesis (i) and Lemma 2.2, we have

$$
\begin{aligned}
\varphi(u) & =\frac{1}{2}\|u\|^{2}+\frac{1}{2} \alpha_{1} u^{2}(0)+\frac{1}{2} \alpha_{2} u^{2}(T)-\int_{0}^{T} F(t, u(t)) d t-\sum_{j=1}^{p} \int_{0}^{u\left(t_{j}^{-}\right)} I_{j}(s) d s \\
& \geq \frac{1}{2}\|u\|^{2}-\sum_{j=1}^{p} \int_{0}^{u\left(t_{j}^{-}\right)} I_{j}(s) d s \\
& \geq \frac{1}{2}\|u\|^{2}-\sum_{j=1}^{p} \int_{0}^{\|u\|_{\infty}}\left(a_{j}|x|^{\eta_{j}}+b_{j}\right) d x \\
& \geq \frac{1}{2}\|u\|^{2}-\sum_{j=1}^{p}\left(\frac{a_{j}}{\eta_{j}+1}\|u\|_{\infty}^{\eta_{j}+1}+b_{j}\|u\|_{\infty}\right) \\
& \geq \frac{1}{2}\|u\|^{2}-\sum_{j=1}^{p}\left(\frac{a_{j} c^{\eta_{j}+1}}{\eta_{j}+1}\|u\|^{\eta_{j}+1}+b_{j} c\|u\|\right),
\end{aligned}
$$

which implies that $\varphi$ is coercive since $\eta_{j} \in(0,1)$ for $j=1,2, \ldots, p$.

Therefore by the least action principle [27, Theorem 1.1], we obtain that $c^{\prime}=\inf _{X} \varphi(u)$ is a critical value of $\varphi$, this means that there exists $\tilde{u} \in X$ such that $\varphi(\tilde{u})=c^{\prime}$.

Finally, we show that $\tilde{u}$ is nontrivial.

In (3.24), if we choose positive constant $k$ such that $k<1 / t_{1}$, then $\bar{u}(t) \in[0,1]$ for $t \in[0,1]$, and for any $r \in(0,1)$, by the assumption (ii) and with same calculation of (3.25), we have

$$
F(t, r \bar{u}) \geq-\frac{a_{0}}{\eta_{0}+1}\|r \bar{u}\|_{\infty}^{\eta_{0}+1}, \quad \int_{0}^{r \bar{u}\left(t_{j}^{-}\right)} I_{j}(s) d s \geq \frac{\tilde{a}_{j}}{v_{j}+1}\left|r \bar{u}\left(t_{j}^{-}\right)\right|^{v_{j}+1} .
$$

Then

$$
\begin{aligned}
& \varphi(r \bar{u}) \\
& \quad=\frac{1}{2}\|r \bar{u}\|^{2}+\frac{1}{2} \alpha_{1}(r \bar{u}(0))^{2}+\frac{1}{2} \alpha_{2}(r \bar{u}(T))^{2}-\int_{0}^{T} F(t, r \bar{u}) d t-\sum_{j=1}^{p} \int_{0}^{r \bar{u}\left(t_{j}^{-}\right)} I_{j}(s) d s \\
& \quad \leq \frac{r^{2}}{2}\left(\|\bar{u}\|^{2}+\alpha_{1}(\bar{u}(0))^{2}+\alpha_{2}(\bar{u}(T))^{2}\right)+\frac{a_{0} T c^{\eta_{0}+1}}{\eta_{0}+1}\|\bar{u}\|^{\eta_{0}+1} r^{\eta_{0}+1}-\sum_{j=1}^{p} \frac{\tilde{a}_{j}}{v_{j}+1}\left|\bar{u}\left(t_{j}^{-}\right)\right|^{v_{j}+1} r^{\nu_{j}+1} .
\end{aligned}
$$

For $r>0$ small enough, we have that $\varphi(r \bar{u})<0$ since $0<v_{j}<\min \left\{\eta_{0}, 1\right\}$. Hence, $\varphi(\widetilde{u})=$ $\inf _{X} \varphi(u)<0$, and by Remark 3.5, $\tilde{u}$ is a nontrivial solution generated by impulses. 
Example 3.8. Let $T=1, t_{1} \in(0,1)$, consider the system:

$$
\begin{gathered}
-u^{\prime \prime}(t)+u(t)=f(t, u(t)), \quad t \in[0,1] \backslash\left\{t_{1}\right\}, \\
u\left(t_{1}^{-}\right)-u\left(t_{1}^{+}\right)=\frac{1}{3} u\left(t_{1}^{-}\right), \\
u^{\prime}\left(t_{1}^{-}\right)-u^{\prime}\left(t_{1}^{+}\right)=u^{3}\left(t_{1}^{-}\right)-\frac{1}{3} u^{\prime}\left(t_{1}^{+}\right), \\
u(0)-u^{\prime}(0)=0, \quad u(1)+u^{\prime}(1)=0 .
\end{gathered}
$$

Compare with problem $(\mathrm{P}), A_{1}=1 / 3, I_{1}(u)=u^{3}$.

If we choose $f(t, u)=u^{p / q}$, where $p$ and $q$ are odd numbers and $p / q>1$, then the conditions of Theorem 3.3 are satisfied. So, the impulsive boundary value problem (3.32) has an infinite number of nontrivial solutions.

Example 3.9. Consider (3.32) with $f(t, u)$ defined by

$$
f(t, u)= \begin{cases}-u e^{u}, & u \leq 1 \\ -e, & u>1\end{cases}
$$

If we choose $a=e, \eta=1 / 2$, the assumptions of Theorem 3.6 are satisfied, then the impulsive boundary value problem (3.32) has at least one nontrivial solution generated by impulses.

Example 3.10. Consider the following problem:

$$
\begin{gathered}
-u^{\prime \prime}(t)+u(t)=f(t, u(t)), \quad t \in[0,1], \\
\alpha u(0)-\beta u^{\prime}(0)=0, \quad \gamma u(1)+\sigma u^{\prime}(1)=0 .
\end{gathered}
$$

If we choose $f(t, u)=-t u^{m / n}-u^{p / q}$, where $m, n, p$, and $q$ are odd numbers, then $u f(t, u) \leq 0$, from Lemma 3.4, we know that (3.34) has no nontrivial solution.

Example 3.11. Let $T=1, t_{1} \in(0,1)$, we consider the following problem:

$$
\begin{gathered}
-u^{\prime \prime}(t)+u(t)=-u^{1 / 9}(t), \quad t \in[0,1] \backslash\left\{t_{1}\right\}, \\
u\left(t_{1}^{-}\right)-u\left(t_{1}^{+}\right)=\frac{1}{3} u\left(t_{1}^{-}\right), \\
u^{\prime}\left(t_{1}^{-}\right)-u^{\prime}\left(t_{1}^{+}\right)=u^{1 / 3}\left(t_{1}^{-}\right)-\frac{1}{3} u^{\prime}\left(t_{1}^{+}\right), \\
u(0)-u^{\prime}(0)=0, \quad u(1)+u^{\prime}(1)=0,
\end{gathered}
$$

here $f(t, u)=-u^{1 / 9}, A_{1}=1 / 3, I_{1}(u)=u^{1 / 3}$. In view of $u f(t, u)=-u^{10 / 9} \leq 0$ and Lemma 3.4, we get that the above problem without impulsive, that is, the problem:

$$
-u^{\prime \prime}(t)+u(t)=-u^{1 / 9}(t), \quad t \in[0,1], \quad u(0)-u^{\prime}(0)=0, \quad u(1)+u^{\prime}(1)=0
$$


has no nontrivial solution. In addition, it is easy to verify that the hypotheses of Theorem 3.7 are fulfilled. Thus, via Theorem 3.7, the impulsive boundary value problem (3.35) has at least one nontrivial solutions generated by impulses.

\section{Acknowledgments}

The research of H. R. Sun was supported by NSF of China (10801065), FRFCU (lzujbky-201143, lzujbky-2012-k25) and SRF for ROCS, and SEM. The research of J. J. Nieto was partially supported by Ministerio de Ciencia e lnnovación and FEDER, Project MTM2010-15314.

\section{References}

[1] M. Benchohra, J. Henderson, and S. Ntouyas, Impulsive Differential Equations and Inclusions, vol. 2 of Contemporary Mathematics and Its Applications, Hindawi Publishing Corporation, New York, NY, USA, 2006.

[2] K. G. Dishlieva, "Differentiability of solutions of impulsive differential equations with respect to the impulsive perturbations," Nonlinear Analysis, vol. 12, no. 6, pp. 3541-3551, 2011.

[3] W. M. Haddad, V. Chellaboina, and S. G. Nersesov, Impulsive and Hybrid Dynamical Systems, Princeton Series in Applied Mathematics, Princeton University Press, Princeton, NJ, USA, 2006.

[4] R. I. Leine, U. Aeberhard, and C. Glocker, "Hamilton's principle as variational inequality for mechanical systems with impact," Journal of Nonlinear Science, vol. 19, no. 6, pp. 633-664, 2009.

[5] X. Lin and D. Jiang, "Multiple positive solutions of Dirichlet boundary value problems for second order impulsive differential equations," Journal of Mathematical Analysis and Applications, vol. 321, no. 2, pp. 501-514, 2006.

[6] A. M. Samoilenko and N. A. Perestyuk, Impulsive Differential Equations, vol. 14 of World Scientific Series on Nonlinear Science. Series A: Monographs and Treatises, World Scientific Publishing, River Edge, NJ, USA, 1995.

[7] J. Xiao, J. J. Nieto, and Z. Luo, “Multiple positive solutions of the singular boundary value problem for second-order impulsive differential equations on the half-line," Boundary Value Problems, vol. 2010, Article ID 281908, 13 pages, 2010.

[8] S. T. Zavalishchin and A. N. Sesekin, Dynamic Impulse Systems: Theory and Applications, vol. 394 of Mathematics and its Applications, Kluwer Academic, Dordrecht, The Netherlands, 1997.

[9] L. Zhang and Z. Teng, "N-species non-autonomous Lotka-Volterra competitive systems with delays and impulsive perturbations," Nonlinear Analysis, vol. 12, no. 6, pp. 3152-3169, 2011.

[10] L. Bai and B. Dai, "An application of variational method to a class of Dirichlet boundary value problems with impulsive effects," Journal of the Franklin Institute, vol. 348, no. 9, pp. 2607-2624, 2011.

[11] J. J. Nieto, "Variational formulation of a damped Dirichlet impulsive problem," Applied Mathematics Letters, vol. 23, no. 8, pp. 940-942, 2010.

[12] J. J. Nieto and D. O'Regan, "Variational approach to impulsive differential equations," Nonlinear Analysis, vol. 10, no. 2, pp. 680-690, 2009.

[13] J. Sun and H. Chen, "Variational method to the impulsive equation with Neumann boundary conditions," Boundary Value Problems, vol. 2009, Article ID 316812, 17 pages, 2009.

[14] J. Sun and H. Chen, "Multiplicity of solutions for a class of impulsive differential equations with Dirichlet boundary conditions via variant fountain theorems," Nonlinear Analysis, vol. 11, no. 5, pp. 4062-4071, 2010.

[15] J. Sun, H. Chen, and J. J. Nieto, “On ground state solutions for some non-autonomous SchrödingerPoisson systems," Journal of Differential Equations, vol. 252, pp. 3365-3380, 2012.

[16] J. Sun, H. Chen, J. J. Nieto, and M. Otero-Novoa, "The multiplicity of solutions for perturbed secondorder Hamiltonian systems with impulsive effects," Nonlinear Analysis, vol. 72, no. 12, pp. 4575-4586, 2010.

[17] Y. Tian and W. Ge, "Multiple positive solutions for a second order Sturm-Liouville boundary value problem with a $p$-Laplacian via variational methods," The Rocky Mountain Journal of Mathematics, vol. 39, no. 1, pp. 325-342, 2009.

[18] Y. Tian and W. Ge, "Applications of variational methods to boundary-value problem for impulsive differential equations," Proceedings of the Edinburgh Mathematical Society. Series 2, vol. 51, no. 2, pp. 509-527, 2008. 
[19] Y. Tian and W. Ge, "Variational methods to Sturm-Liouville boundary value problem for impulsive differential equations," Nonlinear Analysis, vol. 72, no. 1, pp. 277-287, 2010.

[20] Y. Tian and W. Ge, "Multiple solutions of impulsive Sturm-Liouville boundary value problem via lower and upper solutions and variational methods," Journal of Mathematical Analysis and Applications, vol. 387, no. 2, pp. 475-489, 2012.

[21] J. Xiao and J. J. Nieto, "Variational approach to some damped Dirichlet nonlinear impulsive differential equations," Journal of the Franklin Institute, vol. 348, no. 2, pp. 369-377, 2011.

[22] J. Xiao, J. J. Nieto, and Z. Luo, "Multiplicity of solutions for nonlinear second order impulsive differential equations with linear derivative dependence via variational methods," Communications in Nonlinear Science and Numerical Simulation, vol. 17, no. 1, pp. 426-432, 2012.

[23] H. Zhang and Z. Li, "Variational approach to impulsive differential equations with periodic boundary conditions," Nonlinear Analysis, vol. 11, no. 1, pp. 67-78, 2010.

[24] H. Zhang and Z. Li, "Periodic and homoclinic solutions generated by impulses," Nonlinear Analysis, vol. 12, no. 1, pp. 39-51, 2011.

[25] Z. Zhang and R. Yuan, "An application of variational methods to Dirichlet boundary value problem with impulses," Nonlinear Analysis, vol. 11, no. 1, pp. 155-162, 2010.

[26] J. Zhou and Y. Li, "Existence and multiplicity of solutions for some Dirichlet problems with impulsive effects," Nonlinear Analysis, vol. 71, no. 7-8, pp. 2856-2865, 2009.

[27] J. Mawhin and M. Willem, Critical Point Theory and Hamiltonian Systems, vol. 74 of Applied Mathematical Sciences, Springer, New York, NY, USA, 1989. 


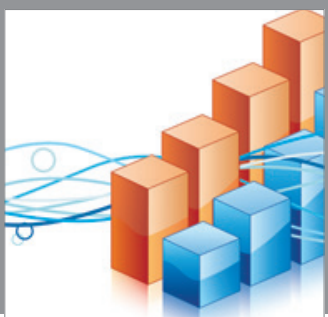

Advances in

Operations Research

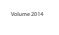

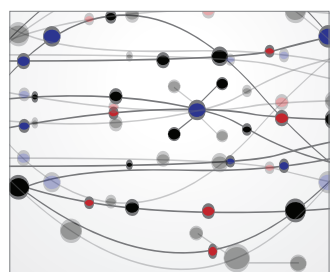

\section{The Scientific} World Journal
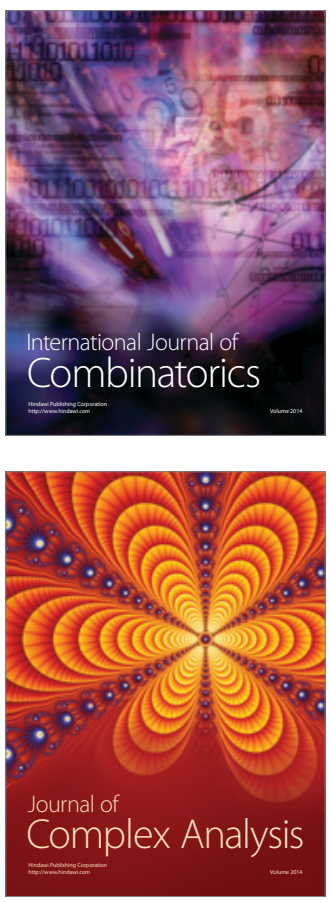

International Journal of

Mathematics and

Mathematical

Sciences
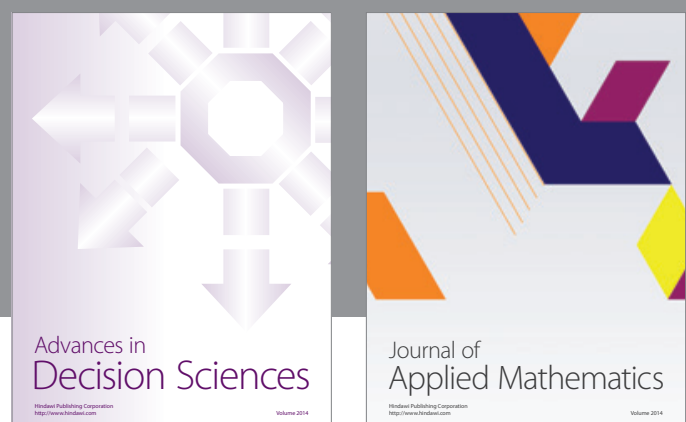

Journal of

Applied Mathematics
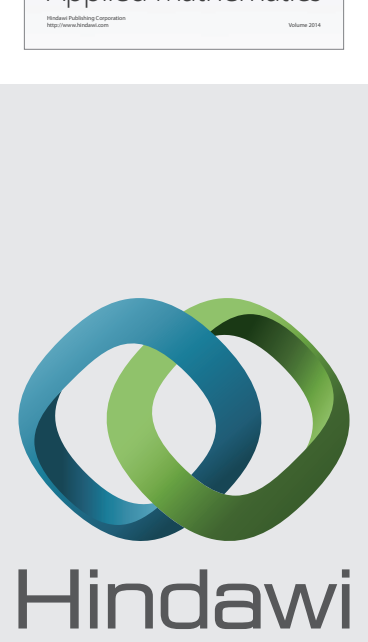

Submit your manuscripts at http://www.hindawi.com
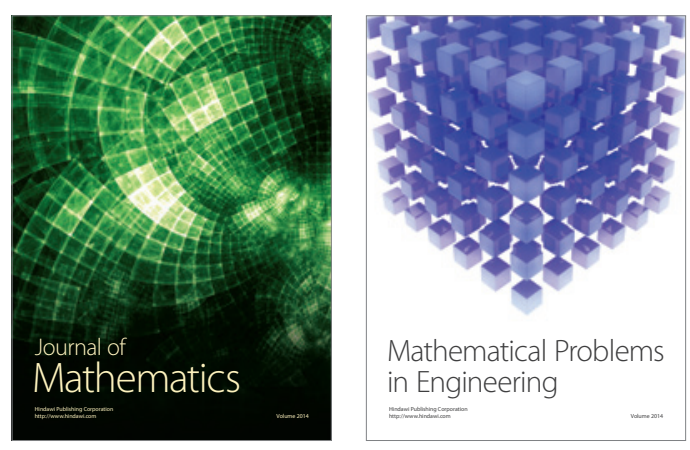

Mathematical Problems in Engineering
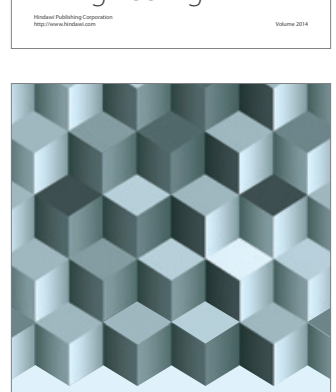

Journal of

Function Spaces
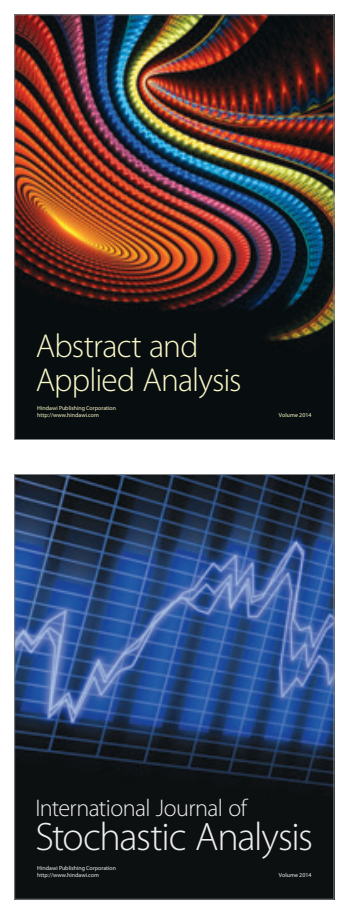

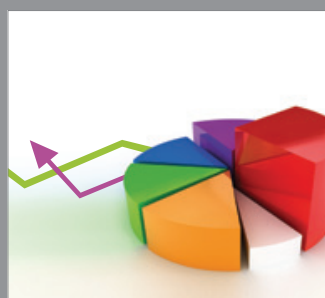

ournal of

Probability and Statistics

Promensencen
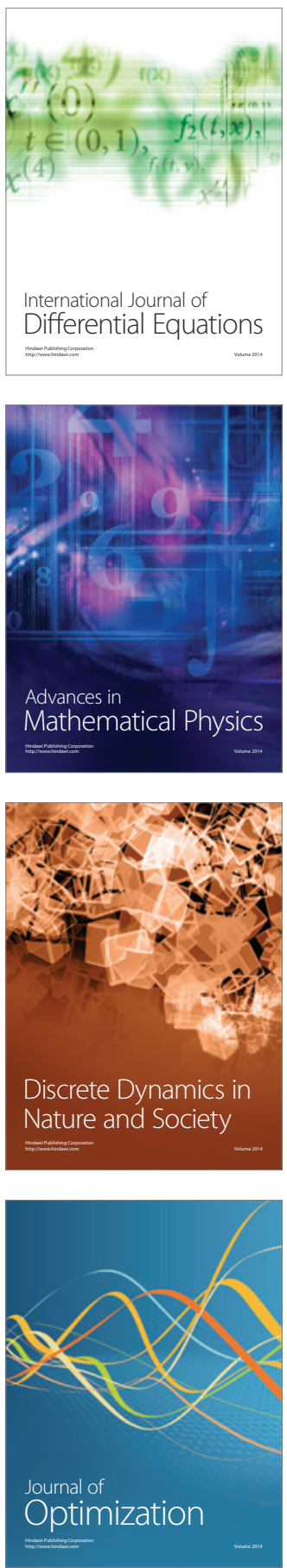